\title{
Facilitation of Serotonin Signaling by SSRIs is Attenuated by Social Isolation
}

\author{
Elyse C Dankoski', Kara L Agster ${ }^{2,3}$, Megan E Fox ${ }^{4}$, Sheryl S Moy ${ }^{2,3}$ and R Mark Wightman*,1,4,5 \\ 'Curriculum in Neurobiology, University of North Carolina at Chapel Hill, Chapel Hill, NC, USA; ${ }^{2}$ Carolina Institute for Developmental Disabilities, \\ University of North Carolina at Chapel Hill, Chapel Hill, NC, USA; 'Department of Psychiatry, University of North Carolina at Chapel Hill, Chapel \\ Hill, NC, USA; ${ }^{4}$ Department of Chemistry, University of North Carolina at Chapel Hill, Chapel Hill, NC, USA; ${ }^{5}$ Center for Neuroscience, University \\ of North Carolina at Chapel Hill, Chapel Hill, NC, USA
}

\begin{abstract}
Hypofunction of the serotonergic system is often associated with major depression and obsessive compulsive disorder (OCD). Selective serotonin reuptake inhibitors (SSRIs) are commonly prescribed to treat these disorders, and require 3-6 weeks of chronic treatment before improvements in the symptoms are observed. SSRIs inhibit serotonin's transporter, and in doing so, increase extracellular serotonin concentrations. Thus, efficacy of SSRIs likely depends upon the brain's adaptive response to sustained increases in serotonin levels. Individual responsiveness to SSRI treatment may depend on a variety of factors that influence these changes, including ongoing stress. Social isolation is a passive, naturalistic form of chronic mild stress that can model depression in rodents. In this study, we examined how 20-day treatment with the SSRI citalopram (CIT) alters marble-burying (MB), open field behavior, and serotonin signaling in single- vs pair-housed animals. We used in vivo voltammetry to measure electrically evoked serotonin, comparing release rate, net overflow, and clearance. Pair-housed mice were significantly more responsive to CIT treatment, exhibiting reduced MB and facilitation of serotonin release that positively correlated with the frequency of electrical stimulation. These effects of CIT treatment were attenuated in singlehoused mice. Notably, although CIT treatment enhanced serotonin release in pair-housed mice, it did not significantly alter uptake rate. In summary, we report that chronic SSRI treatment facilitates serotonin release in a frequency-dependent manner, and this effect is blocked by social isolation. These findings suggest that the efficacy of SSRIs in treating depression and OCD may depend on ongoing stressors during treatment.

Neuropsychopharmacology (2014) 39, 2928-2937; doi:I0.1038/npp.20I4.162; published online 23 July 2014
\end{abstract}

\section{INTRODUCTION}

Disorders involving depression and anxiety, including major depression and obsessive compulsive disorder (OCD), have high rates of comorbidity and are frequently attributed to hypofunction of the serotonergic system (Bespalov et al, 2010; Jacobsen et al, 2012). The most commonly prescribed medications in the treatment of depression and OCD are selective serotonin reuptake inhibitors (SSRIs), which inhibit serotonin's transporter (SERT). Acute SERT inhibition increases brain-wide serotonin levels and depresses firing of serotonergic neurons (Blier and de Montigny, 1998a). Clinically, 3-6 weeks of continuous SSRI treatment are required before symptoms of depression and OCD begin to improve (de Montigny et al, 1992). This gap between treatment onset and therapeutic response suggests that the mechanism by which SSRIs exert antidepressive and

*Correspondence: Dr RM Wightman, Department of Chemsitry, University of North Carolina at Chapel Hill, Campus Box \#3290, Venable Hall, Chapel Hill, NC 27599, USA, Tel: + I 919962 1472, Fax: + 919962 2388, E-mail: rmw@unc.edu

Received 18 March 2014; revised 25 June 2014; accepted 26 June 20।4; accepted article preview online I July 2014 anxiolytic effects is not only due to an increase in ambient serotonin, but also due to an adaptive response in neural systems to sustained increases in serotonin levels. Indeed, a multitude of changes in gene expression, receptor sensitivity, neurochemical signaling, and functional activity coincide with the therapeutic window of SSRI therapy (Bespalov et al, 2010; Blier and de Montigny, 1998a; de Montigny et al, 1992).

Although it is common to treat depression and OCD with SSRIs, the effectiveness of the treatment is also notoriously variable between individuals. Reports estimate that over $60 \%$ of depressed individuals and $40-60 \%$ of individuals with OCD are resistant to improvement while on SSRI therapy (Brandl et al, 2012; Gaynes et al, 2008). Depressed individuals with comorbid anxiety disorders have poorer outcomes on all types of antidepressant medication when compared with non-anxious depressed individuals (Fava et al, 2008). The serotonergic system is highly sensitive to stress, and genetic variability in its function contributes to stress resilience and responsiveness to SSRI therapy (Chaouloff et al, 1999; Oreland et al, 2010; Uher, 2011). Clinical research suggests that ongoing stress, including the absence of social support networks, can contribute to treatment-resistant depression and anxiety (Karelina and 
DeVries, 2011). Thus, improving pharmacotherapies for depression and OCD will likely require an understanding of how stress and anxiety interact with SSRI treatments. Social isolation is a form of chronic mild stress that replicates naturalistic environmental stressors for rodents, and has been suggested as an animal model for depressive disorders (Nestler and Hyman, 2010; Wiborg, 2013). In mice, social isolation significantly upregulates the function of the hypothalamic-pituitary-adrenal (HPA) axis, altering levels of glucocorticoid (CORT) and corticotropin releasing factor (CRF) in various brain regions (Backstrom and Winberg, 2013; Blanchard et al, 2001; Valzelli, 1973). The HPA axis also regulates serotonin signaling and the effects of antidepressants (Barden, 1996, 2004; Pariante and Miller, 2001). Understanding how ongoing social isolation alters serotonin signaling in mice, particularly in response to SSRIs, may provide clues about how stress influences treatmentresistant depression and OCD.

SSRI treatment changes serotonin metabolism, receptors, and transporters in ways that could facilitate serotonergic activity. However, prior to this work, the effects of chronic SSRIs on endogenous in vivo serotonin release and uptake have not been reported. We used fast-scan cyclic voltammetry (FSCV) to monitor serotonin signaling and alterations in its dynamics induced by chronic citalopram (cCIT). FSCV is an electrochemical method with sufficient chemical resolution necessary to identify serotonin (Hashemi et al, 2009), and sufficient temporal resolution to resolve the processes of release from neuronal uptake (Hashemi et al, 2012). We have established that FSCV can monitor serotonin release in vivo, specifically in the substantia nigra pars reticulata (SNpr), which receives a dense serotonergic projection from the dorsal raphe nucleus (DRN). Here, we use FSCV to evaluate the effects of 20-day CIT exposure on serotonin signaling in single-and pair-housed mice. We found robust behavioral and neurochemical differences in the effects of SSRI treatment between these groups.

\section{MATERIALS AND METHODS}

\section{Animals}

All experiments were performed in compliance with the University of North Carolina at Chapel Hill (UNC) Institutional Animal Care and Use Committee. Subjects were C57BL/6J male mice (4-5 weeks in age and 20-27g upon arrival from Jackson Laboratory, Bar Harbor, ME), initially housed in groups of four. After 1 week of acclimation, 40 mice were housed individually, and 36 mice were housed in pairs. All subjects were kept on a 12-hour-day/light cycle and given ad libitum access to food and water.

\section{cCIT Treatment}

To eliminate stress contribution from daily injections, CIT hydrobromide (Roxane Laboratories) or vehicle (VEH) was administered via water bottles for 20 days. Briefly, water consumption was monitored for 7 days to establish a baseline for each subject or pair of subjects. The concentration of CIT added to water during the treatment period was adjusted to $15 \mathrm{mg} / \mathrm{kg} /$ day (per oral) based on subject weights and previous-day consumption volumes through- out the treatment. CIT was obtained in a sweetened oral solution formulation; therefore, $\mathrm{VEH}$-treated animals received water containing D-Sorbitol ( $1.68 \mathrm{~g} / 5 \mathrm{ml}$; Sigma-Aldrich) that matched the concentration in CIT solution. Using this drug delivery method, individual differences in liquid consumption are difficult to ascertain in pair-housed mice; however, close inspection of drinking patterns in pair- vs single-housed mice suggest no individual aberrations in liquid consumption. Comparisons of overall consumption can be found in Supplementary Figure 1A and B. On day 21, treatment was discontinued, and mice were given bottles containing only water. A 24-hour withdrawal period prior to voltammetric experiments was selected based on pharmacokinetic analysis of CIT clearance in mice and implemented prior to voltammetric experiments in order to look at the effects of cCIT treatment in absence of CIT (Fredricson Overo, 1982).

\section{Marble-Burying Assay}

On day 21,20 marbles were arranged in a $4 \times 5$ grid in a $(34 \times 20.5 \times 13.5 \mathrm{~cm})$ cage containing $5 \mathrm{~cm}$ of bedding. Mice were placed inside the cage for $30 \mathrm{~min}$, after which the number of buried marbles ( $<2 / 3$ visible above bedding) were counted.

\section{Open Field Test}

On day $21,4-5 \mathrm{~h}$ after the marble-burying (MB) assay, mice were evaluated for activity in an open field (OF) chamber $(41 \times 41 \times 30 \mathrm{~cm})$ crossed by a grid of photobeams (VersaMax system, AccuScan Instruments). Counts were taken of the number of photobeams broken during the trial in 5-min intervals across $1 \mathrm{~h}$, with separate measures for total distance traveled, fine movements (repeated breaking of the same set of photobeams), and rearing movements (vertical activity). Time spent in the center region of the OF was also recorded.

\section{Detection of Evoked 5-HT Release}

$24 \mathrm{~h}$ after withdrawal from CIT or VEH solutions, mice were evaluated for CIT-induced changes in electrically evoked serotonin release, using previously described methods (Hashemi et al, 2009). See Supplementary Methods for more details.

\section{Drugs and Reagents}

CIT hydrobromide (obtained as oral solution, $10 \mathrm{mg} / 5 \mathrm{ml}$; Roxane Laboratories) was diluted with water for chronic treatment. CIT hydrobromide (for acute treatment), nomifensine maleate salt, and urethane were dissolved in sterile saline (0.9\%). Intraperitoneal-injected drugs (for acute treatments) were administered at a volume of $0.01 \mathrm{ml} / \mathrm{g}$ body weight. All reagents were purchased from SigmaAldrich (St Louis, MO) and used as received.

\section{Data Analysis}

Results are average values \pm SEM. Behavioral data were analyzed using a two-way or repeated measures ANOVA 
with Bonferroni post hoc analyses. Trends in maximum evoked amplitude $\left([5-\mathrm{HT}]_{\max }\right)$ were analyzed using a repeated measures ANOVA that compared the effects of treatment between each housing group (single or pair), using Sidak multiple comparisons post hoc analysis.

Release rate was calculated by fitting lines to the rising phase of serotonin release for each stimulation frequency (for each subject). To evaluate uptake, clearance half-life $\left(t_{1 / 2}\right)$ was calculated in Clampfit (Molecular Devices, LLC, USA) as the decay time between 100 and $50 \%$ evoked concentration for each subject and averaged. Because evoked serotonin concentrations are significantly lower than the $K_{\mathrm{M}}$ for SERT (170 nM) (Shaskan and Snyder, 1970), MichaelisMenten kinetic analyses cannot provide unequivocal distinctions of $K_{\mathrm{M}}$ and $V_{\max }$. In instances where uptake is unsaturated, it follows exponential, first-order decay, and its half-life is therefore independent of peak concentration. Moreover, $t_{1 / 2}$ has been shown to highly correlate with changes in apparent $K_{\mathrm{M}}$ (Yorgason et al, 2011). Thus, treating uptake as a first-order process characterized by $t_{1 / 2}$ is the most appropriate analysis for these data. Increases in this decay time reflect decreases in clearance rate. Net overflow was calculated in Clampfit using the integral under the curve for the first $15 \mathrm{~s}$ following the stimulation event for each stimulation frequency (for each subject). Net overflow analyses have also been shown to correlate well with kinetic models of neurotransmitter release (Yorgason et al, 2011). Groups were compared using a two-way ANOVA with Tukey's multiple comparisons post hoc analysis.

To verify whether evoked serotonin release was frequencydependent, $[5-\mathrm{HT}]_{\max }$ evoked at each stimulation frequency was normalized within subjects to $[5-\mathrm{HT}]_{\max }$ evoked by $20 \mathrm{~Hz}$ stimulations. A line was fit to each group of normalized values and a hypothesis test determined whether the regression coefficient (slope) was significantly different from zero. Treatment groups described as having frequency-dependent release meet criteria by having a slope that is significantly different from zero.

\section{RESULTS}

\section{Electrical Stimulation of the DRN Evokes Serotonin Release in the SNpr}

To verify the chemical origin of signals measured using our recording electrode coordinate, we tested the acute effects of a norepinephrine/dopamine uptake inhibitor (nomifensine, $5 \mathrm{mg} / \mathrm{kg}$, i.p.) and an SSRI (CIT, $10 \mathrm{mg} / \mathrm{kg}$, i.p.) on evoked release. In previous studies, a similar nomifensine dosage had robust effects on dopamine signals but was low enough to avoid nonselective effects (Robinson and Wightman, 2004). Likewise, the selected CIT dosage has been used with robust results on serotonin signaling in prior studies (Hashemi et al, 2012; Hashemi et al, 2009). Color plots and traces show serotonin release at baseline (Figure 1a), unaltered $30 \mathrm{~min}$ after nomifensine (Figure $1 \mathrm{~b}$ ), and increased in amplitude and $t_{1 / 2} 30 \mathrm{~min}$ after CIT (Figure 1c). This response pharmacologically confirms that signals measured at this location are serotonergic, without contribution from catecholamines. Serotonin was also identified by its unique cyclic voltammogram (Figure 1e) (Hashemi et al, 2009). Representative histology shows the location of the stereo-
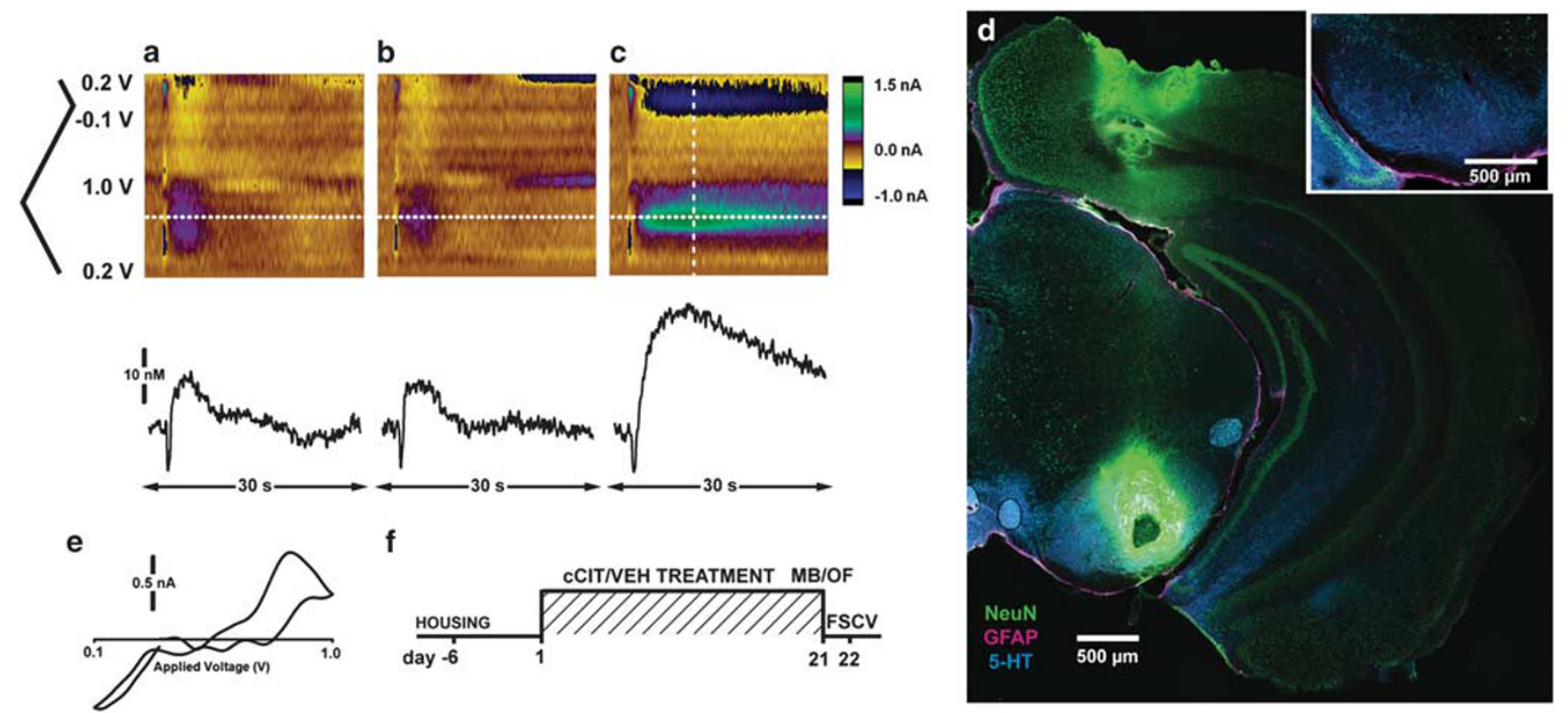

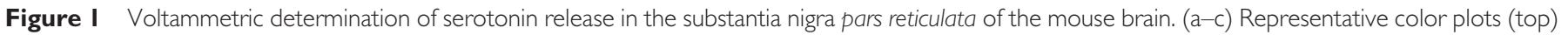

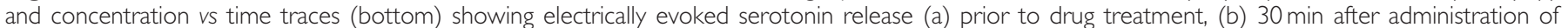

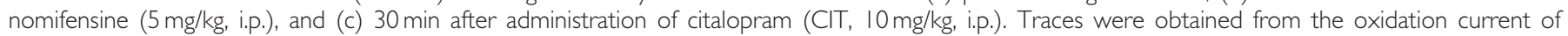

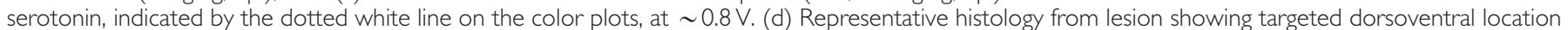

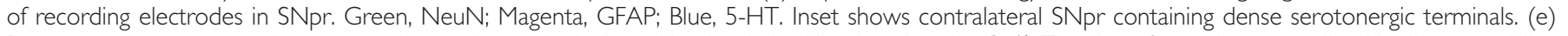

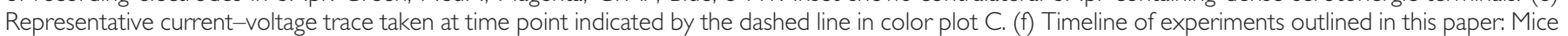

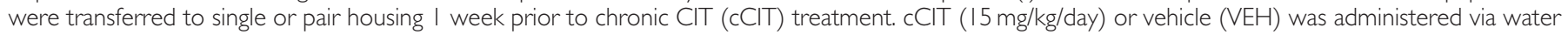

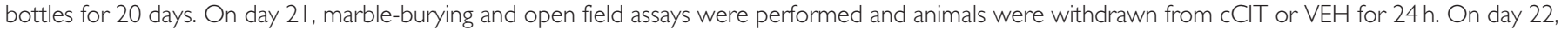
anaesthetized voltammetry experiments were performed. Data shown are means \pm SEM. 
taxic coordinate used for carbon-fiber electrode placement (Figure 1d). Because electrolytic lesions from Nafion-coated carbon-fiber microelectrodes are difficult to locate, we did histology following electrolytic lesions with tungsten wires at the same stereotaxic coordinate. Inset shows the contralateral SNpr, illustrating its dense serotonergic terminal field.

Figure if illustrates the outline of experiments performed in this work. Mice were moved from group housing to either single or pair housing on Day 6, seven days prior to cCIT treatment. During this time period, water consumption for each mouse or pair of mice was monitored to establish the concentration of CIT necessary in chronic treatment. Addition of cCIT or VEH to water bottles began on Day 1 and was monitored and continually adjusted for weight and prior-day consumption throughout 20 days of chronic treatment. On Day 21, MB and OF assays were performed, and cCIT and VEH treatments were replaced with water. On Day 22, mice were anaesthetized and FSCV experiments were performed.

\section{Anxiety-Like Behavior is Reduced by cCIT Treatment}

We selected the treatment period and dosage for cCIT based on prior studies that used oral SSRI administration and found robust neurochemical effects (Davidson and Stamford, 1997, 2000). We used the MB assay, a standard index of anxiety-like behavior, as previous studies had found that chronic SSRI treatment in mice leads to reductions in buried marbles (Arora et al, 2013; Ichimaru et al, 1995). We also performed an OF test for CIT effects on overall activity, as well as additional measures of anxietylike behavior (Jiao et al, 2011; Strekalova et al, 2013).

In line with previous reports (Arora et al, 2013; Ichimaru et al, 1995), we found that cCIT decreased the number of marbles buried (two-way ANOVA, main effect of treatment, $\mathrm{F}(1,72)=9.7, P<0.01)$ (Figure $2 \mathrm{a}$ ). In post hoc analyses, these effects of SSRI treatment were only significant in paired-housed mice $(P<0.05)$. In the OF test, cCIT did not significantly affect general locomotion in either housing group (Figure $2 \mathrm{~b}$ and $\mathrm{c}$ ), confirming previous findings on CIT effects in C57BL/6 mice (Jiao et al, 2011; Strekalova et al, 2013). However, cCIT led to highly significant decreases in time spent in the center region of the OF, in comparison with VEH (Figure 2d; main effect of treatment, $\mathrm{F}(1,72)=19.0, P<0.0001)$. cCIT also led to mild reductions in fine movements, an index of repetitive and stereotyped behavior (Figure 2e; main effect of treatment, $\mathrm{F}(1,72)=10.0, \quad P<0.01)$. Rearing movements, another measure of active exploration in the OF, were decreased by cCIT (Figure 2e; main effect of treatment, $\mathrm{F}(1,68)=9.5$, $P<0.01$ ), although this effect was only significant in post hoc analyses when comparing VEH and cCIT treatment

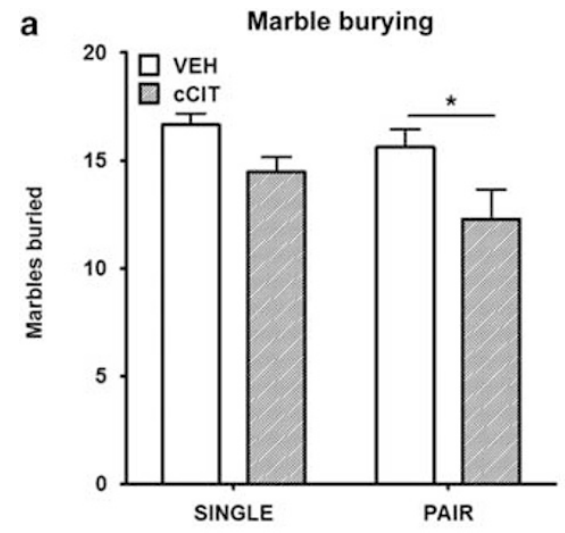

b

Distance traveled (Single)

c

Distance traveled (Pair)
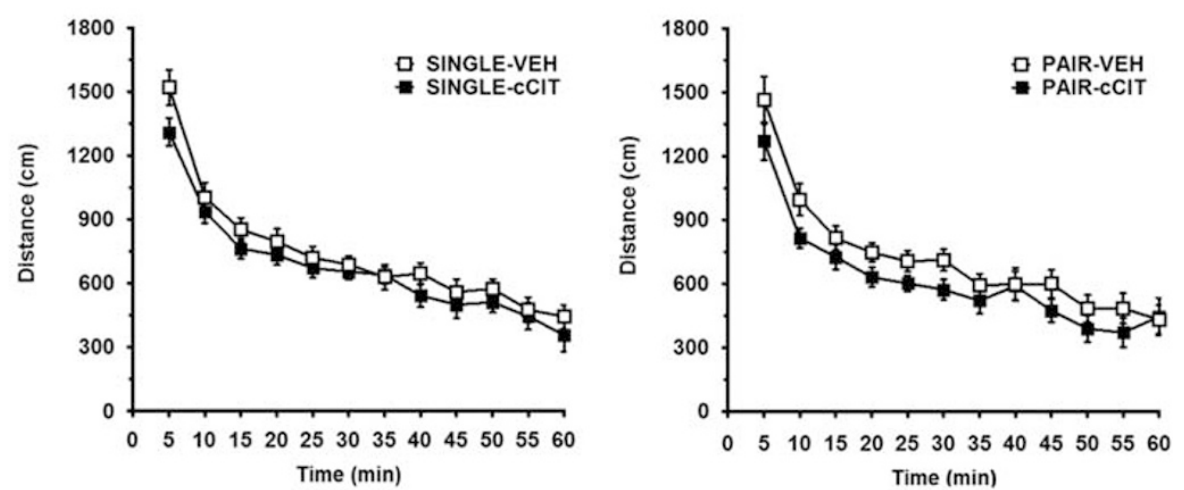

d
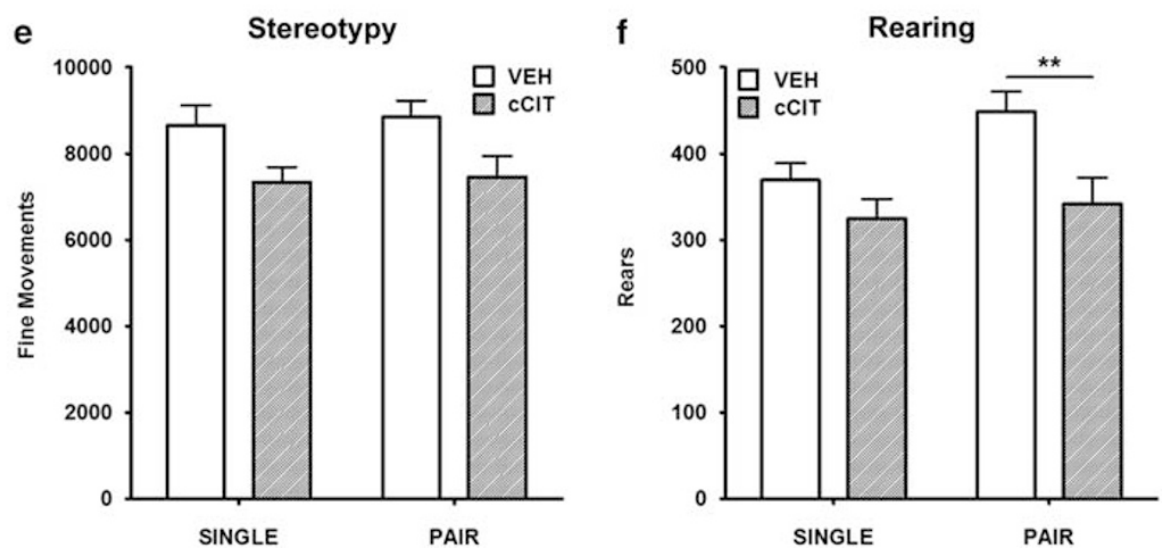

Figure 2 Effects of CCIT or VEH treatment on MB and OF assays in pair- and single-housed mice. (a) Number of marbles (out of 20) buried during a 30 min marble-burying assay. (b and c) Distance traveled across a 60 min OF test. (d) Time spent in center of the OF chamber. (e) Fine movements during OF testing. (f) Incidences of rearing during OF testing. Data shown are means \pm SEM. cCTI, chronic citalopram; OF, open field; MB, marble-burying; $\mathrm{VEH}$, vehicle. Bonferroni post hoc analysis: $* * P<0.01$, $* P<0.05$. 
Table I Maximal [5-HT] Evoked by Stimulations Ranging in Frequency $20-60 \mathrm{~Hz}$

\begin{tabular}{|c|c|c|c|c|c|}
\hline & $20 \mathrm{~Hz}(\mathrm{nM})$ & $30 \mathrm{~Hz}(\mathrm{nM})$ & $40 \mathrm{~Hz}(\mathrm{nM})$ & $50 \mathrm{~Hz}(\mathrm{nM})$ & $60 \mathrm{~Hz}(\mathrm{nM})$ \\
\hline \multicolumn{6}{|l|}{ Baseline } \\
\hline \multicolumn{6}{|l|}{ Single-housed } \\
\hline VEH $(n=6)$ & $16.4 \pm 2.0$ & $15.9 \pm 1.2$ & $16.7 \pm 1.2$ & $16.8 \pm 1.2$ & $17.2 \pm 1.4$ \\
\hline $\mathrm{CCIT}(n=9)$ & $22.3 \pm 2.6$ & $20.4 \pm 1.8$ & $20.8 \pm 2.7$ & $21.5 \pm 2.3$ & $21.9 \pm 2.0$ \\
\hline \multicolumn{6}{|l|}{ Pair-housed } \\
\hline $\mathrm{CCIT}(n=8)$ & $21.8 \pm 2.7$ & $26.1 \pm 3.6$ & $29.3 \pm 4.9$ & $32.3 \pm 4.9 *$ & $32.2 \pm 5.5^{*}$ \\
\hline \multicolumn{6}{|c|}{ Acute citalopram challenge } \\
\hline \multicolumn{6}{|l|}{ Single-housed } \\
\hline $\operatorname{VEH}(n=6)$ & $32.3 \pm 4.3$ & $40.0 \pm 5.5$ & $45.6 \pm 6.2$ & $52.0 \pm 6.7$ & $54.0 \pm 7.1$ \\
\hline
\end{tabular}

Abbreviations: cCIT, chronic citalopram; VEH, vehicle.

Sidak multiple comparisons test.

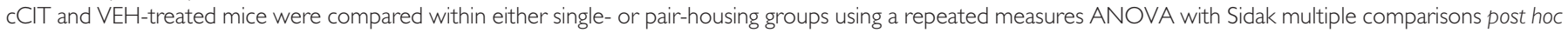
analysis. Starred values denote significant differences between the cCIT value and VEH value immediately above it. $* P<0.05$, $* * P<0.0$ I.

in the pair-housed mice $(P<0.01)$. Overall, cCIT had anxiolytic effects in the $\mathrm{MB}$ assay, but not in the OF test, with more significant effects in the pair-housed mice.

\section{cCIT Increases Serotonin Release in Pair-Housed Mice}

Serotonin release exhibits robust dependence on stimulation frequency when examined in brain slices (Dankoski and Wightman, 2013). This is the result of uptake occurring throughout stimulation pulse trains and has been described using Michaelis-Menten models of neurotransmission (Bunin et al, 1998; Dreyer et al, 2010). Table 1 compares maximal release $\left([5-\mathrm{HT}]_{\max }\right)$ evoked by a range of stimulation frequencies $(20-60 \mathrm{~Hz}, 60$ pulses each). In marked contrast to the slice results, we found that in vivo serotonin signaling was not frequency-dependent under baseline conditions. No significant effect of cCIT or frequency was found when comparing $[5-\mathrm{HT}]_{\max }$ in $\mathrm{VEH}$ vs cCIT-treated single-housed mice. However, cCIT increased $[5-\mathrm{HT}]_{\max }$ in pair-housed mice compared with VEH-treated mice (repeated measures ANOVA, main effect of treatment, $\mathrm{F}(1,12)=5.4, P<0.05)$, with main effects of stimulation frequency $(\mathrm{F}(4,48)=3.9, P<0.01)$, as well as a treatment $\times$ frequency interaction $(\mathrm{F}(4,48)=5.9, P<0.001)$. Post hoc analysis revealed significant differences at 50 and $60 \mathrm{~Hz}$ $(P<0.05)$.

After an acute CIT challenge (Table 1, bottom), the effects of cCIT in pair-housed mice were maintained and intensified (repeated measures ANOVA; main effect of treatment, $\mathrm{F}(1,12)=9.8, P<0.01$; frequency, $\mathrm{F}(4,48)=68.9, P<0.0001$, and treatment $\times$ frequency interaction, $F(4,48)=7.6$, $P<0.0001)$. Post hoc analyses revealed differences between $\mathrm{VEH}$ and cCIT pair-housed mice at stimulus frequencies of $30 \mathrm{~Hz}, 40 \mathrm{~Hz}$ (both at $P<0.05$ ), $50 \mathrm{~Hz}$, and $60 \mathrm{~Hz}$ (both at
$P<0.01)$. Changes in $[5-\mathrm{HT}]_{\max }$ are not correlated with a change in local serotonin content (Supplementary Figure $\mathrm{S} 1 \mathrm{C})$. Therefore, functional changes in neurotransmission must underlie the effects of cCIT treatment.

\section{Increased [5-HT] $]_{\max }$ in cCIT Pair-Housed Mice is Due to Facilitation of Release}

To determine what underlies changes in $[5-\mathrm{HT}]_{\max }$, we analyzed the rate of release, clearance half-life $\left(t_{1 / 2}\right)$, and net overflow in serotonin concentration traces evoked by $60 \mathrm{~Hz}$, $1 \mathrm{~s}$ stimulations (Figure 3). Mice were withdrawn from CIT or VEH for $24 \mathrm{~h}$, sufficient to metabolize all remaining drug (Fredricson Overo, 1982). Thus, serotonin release shown in Figure 3 reflects baseline conditions and is not affected by ongoing uptake inhibition. Figure $3 \mathrm{a}$ and $\mathrm{b}$ shows average concentration traces of serotonin release at $60 \mathrm{~Hz}$ (concentration traces for all stimulation frequencies are in Supplementary Figure S2). cCIT treatment increased release rate (two-way ANOVA, main effect of treatment $\mathrm{F}(1,25)=6.1$, $P<0.05)$, and there was an interaction between treatment and housing $(\mathrm{F}(1,25)=4.6, P<0.05)$ (Figure $3 \mathrm{c}$ ). Post hoc analysis revealed differences in $60 \mathrm{~Hz}$ release rate between pair-housed VEH and cCIT mice $(7.4 \pm 1.1 \mathrm{nM} / \mathrm{s} \quad v s$ $19.2 \pm 3.1 \mathrm{nM} / \mathrm{s}, n=6$ and $n=8$, respectively, $P<0.01)$, but not between single-housed VEH and cCIT mice.

Surprisingly, clearance $\left(t_{1 / 2}\right)$ of evoked serotonin was unaltered by cCIT treatment in either housing group (Figure $3 \mathrm{~d}$ ). This demonstrates that increases in $[5-\mathrm{HT}]_{\max }$ (Table 1) were due to facilitation of release following cCIT treatment. Similar results have been obtained in previous voltammetric studies (Davidson and Stamford, 1997, 2000); however, our result conflicts with the work of Benmansour et al (1999), who found that baseline SERT function was 
a

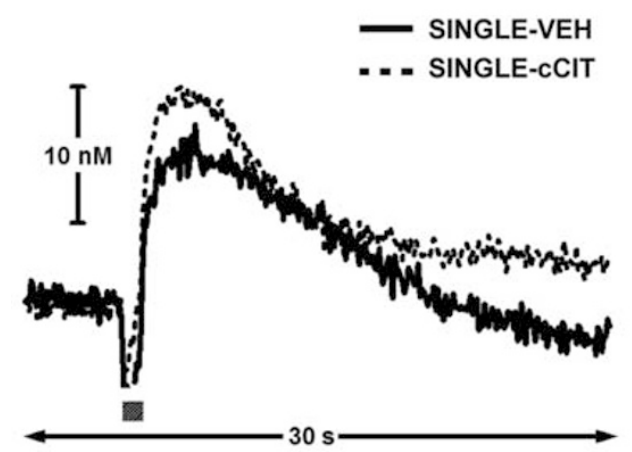

C
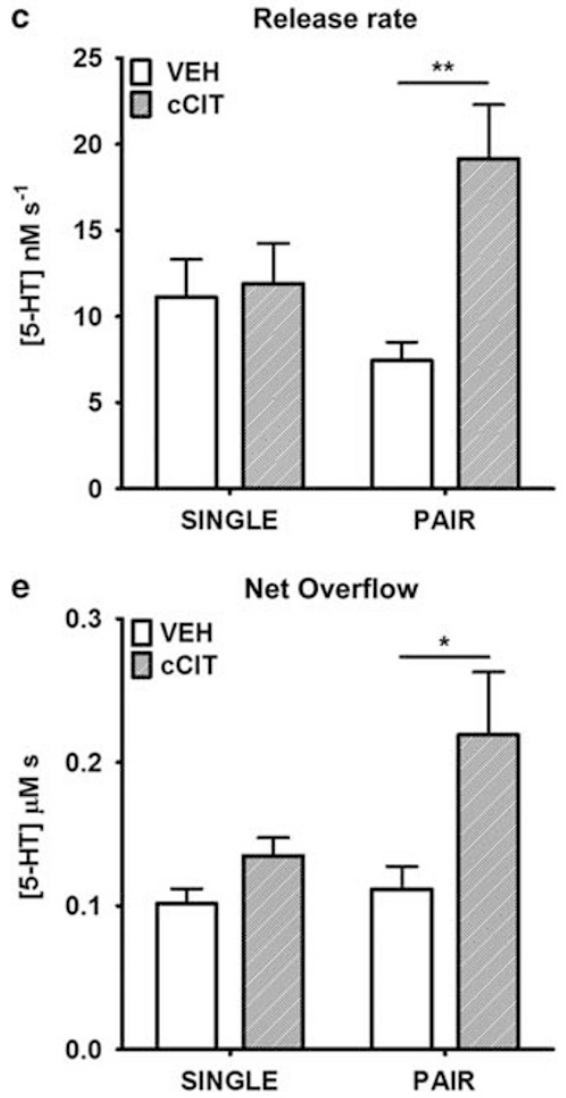

b

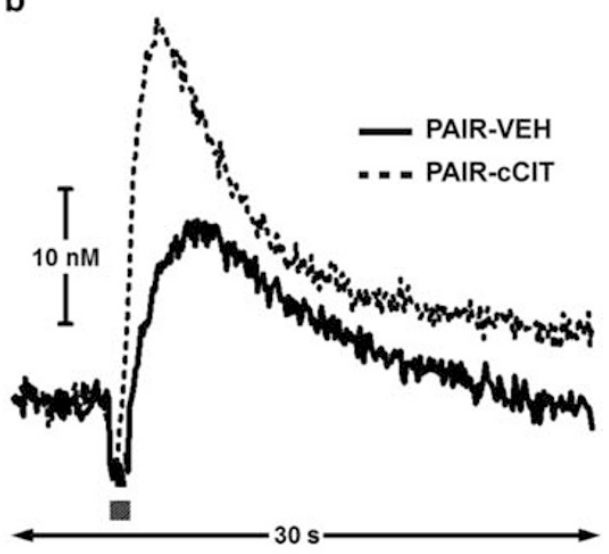

d

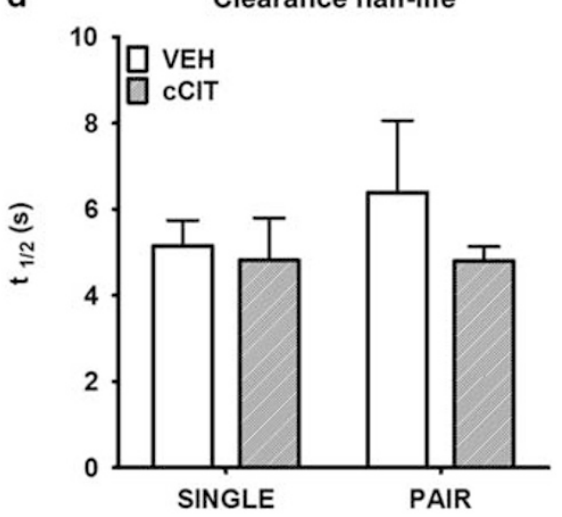

f Frequency dependence

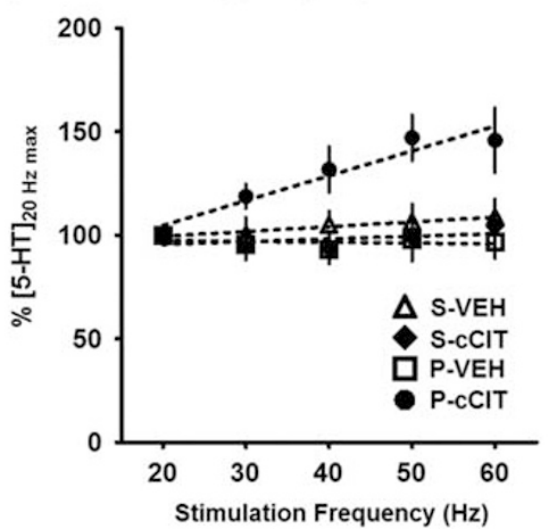

Figure 3 Comparison of serotonin release and uptake evoked by electrical stimulation of the DRN. (a and b) Averaged concentration vs time traces of serotonin release evoked by a $60 \mathrm{~Hz}, 60$-pulse stimulation of the DRN. Shaded bar below trace indicates the duration of stimulation. (c) Mean rate of change in $[5-\mathrm{HT}]$ during the rising phase of release evoked by $60 \mathrm{~Hz}, 60$-pulse stimulation. (d) $t_{1 / 2}$ of evoked serotonin following $60 \mathrm{~Hz}$, 60 -pulse stimulation. (e) Mean net overflow (area under curve) following $60 \mathrm{~Hz}, 60$-pulse stimulations. ( $f$ ) Averages of normalized maximal release amplitudes evoked by $20-60 \mathrm{~Hz}$ stimulations. Dotted lines show best-fit linear regressions for each treatment group. Data shown are means \pm SEM. Tukey's multiple comparisons post hoc analysis: ${ }^{*} * P<0.01$, $* P<0.05$.

impaired by chronic SSRI treatment. Their study was conducted in the hippocampus and measured SERT function using high concentrations of exogenously applied serotonin, critical differences that may explain this discrepancy.

In the present study, cCIT increased net overflow (Figure 3e, two-way ANOVA, $\mathrm{F}(1,25)=6.6, P<0.05)$, and post hoc analysis showed differences between VEH and cCIT treatment in pair-housed $(0.11 \pm 0.02 \mu \mathrm{M} s v s 0.22 \pm 0.04 \mu \mathrm{M}$ s, $n=6$ and $n=8$, respectively, $P<0.05)$ but not singlehoused mice. Taken together, these data show that facilitation of serotonin release is a major contributor to the enhanced serotonergic neurotransmission in pair-housed cCIT mice, whereas uptake is unaffected. 
Because differences in neurotransmission were only apparent in release evoked by higher frequency stimulations, we developed an assay to quantitatively establish frequency dependence, described in Materials and Methods. Only serotonin release in pair-housed cCIT mice met the criteria for frequency dependence (Figure 3f; hypothesis test for regression slope, $\mathrm{F}(1,38)=14.4, P<0.001)$. Frequencydependent facilitation of serotonin release in pair-housed mice is a major outcome of cCIT treatment.

\section{Acute Uptake Inhibition Enhances Facilitation of Serotonin Release in cCIT Pair-Housed Mice}

The above analyses examined how cCIT treatment altered baseline serotonin neurotransmission in the absence of circulating CIT. To understand how cCIT treatment affects serotonin signaling in the presence of CIT, we administered an acute challenge dose of CIT (10 mg/kg i.p.) and compared its effects on evoked serotonin concentrations in single- $v s$ pair-housed and VEH vs cCIT groups. Supplementary Figure 3A-D compares serotonin concentration traces within each group prior to drug administration (baseline) and $30 \mathrm{~min}$ after acute CIT challenge dose (aCIT). In each group, acute CIT treatment significantly enhanced evoked serotonin in a manner that is qualitatively similar to previous reports (Hashemi et al, 2012). Release rate, $t_{1 / 2}$, and net overflow were all increased by the acute CIT challenge as expected.

It is interesting to directly compare serotonin signals under the influence of circulating CIT in VEH- vs cCITtreated animals, as this condition approximates ongoing CIT exposure during clinical antidepressant therapies. Figure 4 examines differences in serotonin signaling between $\mathrm{VEH}$ and cCIT single- and pair-housed mice after $30 \mathrm{~min}$ after the acute CIT challenge was administered. Differences in uptake are evident when comparing average concentration traces evoked by $60 \mathrm{~Hz}, 60$-pulse stimulations (Figure $4 \mathrm{a}$ and b). In all groups, $t_{1 / 2}$ was increased by acute CIT challenge compared with baseline values, but to a lesser extent in cCIT mice (2-way ANOVA, main effect of treatment, $F(1,25)=$ 7.4, $P<0.05$ ) (Figure 4d). This finding concurs with Benmansour et al (1999), who found that SERT function was less responsive to uptake inhibitors after chronic SSRI treatment.

Release rate was altered by cCIT treatment $(\mathrm{F}(1,25)=6.1$, $P<0.05)$ and there was an in interaction between treatment and housing $(\mathrm{F}(1,25)=4.6, P<0.05)$. Post hoc analysis revealed differences in release rate between pair-housed $\mathrm{VEH}$ and cCIT mice $\left(14.3 \pm 2.5 \mathrm{nM} \mathrm{s}^{-1}\right.$ vs $26.9 \pm 3.8 \mathrm{nM} \mathrm{s}^{-1}$, $n=6$ and $n=8$, respectively, $P<0.05)$ but not single-housed $\mathrm{VEH}$ and cCIT mice (Figure 4c). Acute CIT also increased net overflow in cCIT mice (main effect of treatment, $\mathrm{F}(1,25)=6.0, \quad P<0.05)$, with significant main effect of housing $(\mathrm{F}(1,25)=4.50, P<0.05)$ and a treatment $\mathrm{x}$ housing interaction $(\mathrm{F}(1,25)=8.5, P<0.01$ ) (Figure 4e). Post hoc analysis of net overflow revealed differences between $\mathrm{VEH}$ and cCIT in pair-housed mice $(0.53 \pm 0.06 \mu \mathrm{Ms}$ vs $1.13 \pm$ $0.15 \mu \mathrm{Ms}, n=6$ and $n=8$, respectively, $P<0.01)$ and also between single- and pair-housed cCIT mice $(0.57 \pm 0.09 \mu \mathrm{M}$ s vs $1.13 \pm 0.15 \mu \mathrm{M} \mathrm{s}, n=9$ and $n=8$, respectively, $P<0.01)$. The overall increase in release rate and net overflow after acute CIT was expected, owing to SSRI-induced reductions in uptake (Wightman and Zimmerman, 1990). Consistent with this, release rates measured in the various groups of mice varied in proportion to the baseline release rates, and differences were still only observed in pair-housed mice.

We used a hypothesis test to quantitatively confirm frequency dependence following acute CIT. Frequency dependence was determined for all groups (Figure $4 \mathrm{f}$, hypothesis test for regression slope; VEH-single, $\mathrm{F}(1,28)=182.4$, $P<0.0001$; cCIT single, $\mathrm{F}(1,38)=25.9, P<0.0001$; VEHpair, $\mathrm{F}(1,28)=96.2, P<0.0001$; $\mathrm{cCIT}$ pair, $\mathrm{F}(1,43)=84.5$, $P<0.0001)$. Although all groups showed frequency dependence following acute CIT, the magnitude of release at all frequencies was much greater in cCIT-treated paired animals (Table 1 , bottom).

\section{DISCUSSION}

Though SSRIs are commonly prescribed to treat disorders involving depression and anxiety, the mechanisms by which these agents exert therapeutic effects are not clear. Prior studies have examined how chronic SSRI treatment affects the serotonin system, but this is the first to compare effects of SSRI treatment on endogenous, in vivo release dynamics. Furthermore, this study directly addresses the interaction of social environment with serotonergic function in this context. Here, we found distinct differences in the effects of chronic SSRI treatment on serotonin signaling in single- $v s$ pair-housed mice. We describe a novel effect of this treatment-the facilitation of serotonin signaling in a frequency-dependent fashion-and the ability to attenuate this outcome with concurrent social isolation.

Levels of neurotransmitter in the extracellular space are determined by a dynamic equilibrium between release and uptake (Dreyer et al, 2010). Previously, we reported that disruptions of serotonergic equilibria can be fatal, and release and uptake are both tightly controlled to maintain this balance (Hashemi et al, 2012). Further evidence of this control can be seen in Table 1 and Figure 3f: in vivo release in VEH and single-housed cCIT-treated mice is insensitive to the rate of stimulation. In brain slices, serotonin neurons have the capacity for frequency-dependent release (Dankoski and Wightman, 2013), likely because inhibitory feedback from intact serotonergic neurons and other neuromodulatory systems are severed during slice preparation. Spontaneous serotonergic burst firing does occur during distinct behavioral states (Gartside et al, 2000; Jacobs, 1991); however, multiple inhibitory systems preserve the pacemaker-like firing $(1-5 \mathrm{~Hz})$ of serotonergic neurons. For instance, somatodendritic 5-HT1a autoreceptors profoundly limit the firing rate of serotonergic neurons (Blier et al, 1998 b), and the DRN also receives complex feedback from prefrontal cortex (Celada et al, 2001).

Following cCIT treatment, $[5-\mathrm{HT}]_{\max }$ was frequencydependent in pair-housed mice (Table 1). Our analyses showed that this was due to increases in release rate, not changes in $t_{1 / 2}$ (Figure $3 \mathrm{c}$ and $\mathrm{d}$ ). Thus, cCIT increases the responsiveness of serotonergic neurons to higher stimulation frequencies. This could arise from 5-HTla autoreceptor desensitization, a process that is well documented following chronic SSRI exposure and is a critical component of its antidepressant effects (Celada et al, 2013; Ferres-Coy et al, 
a

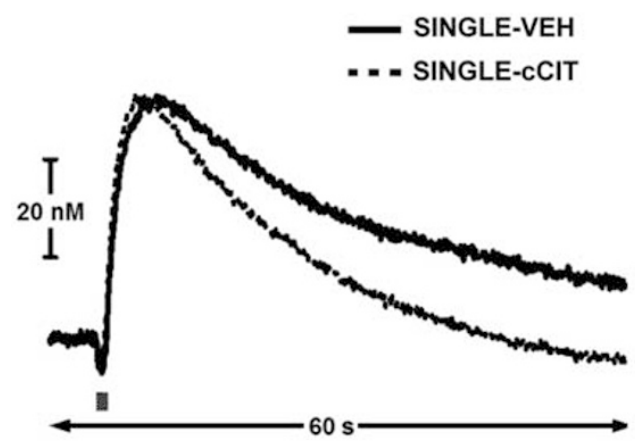

c

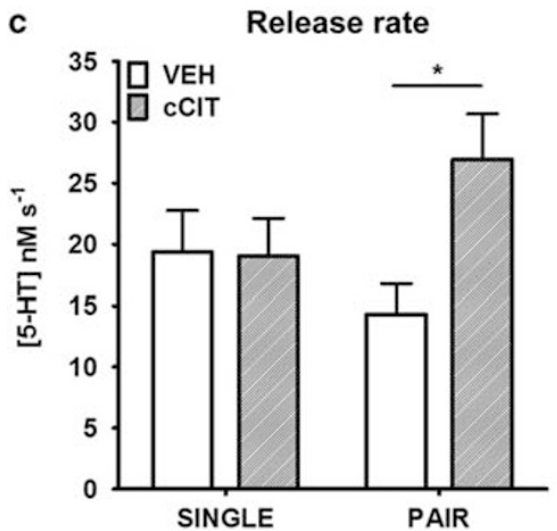

e

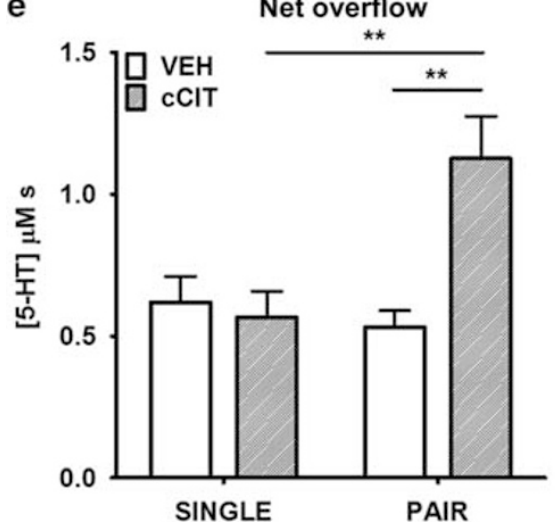

b

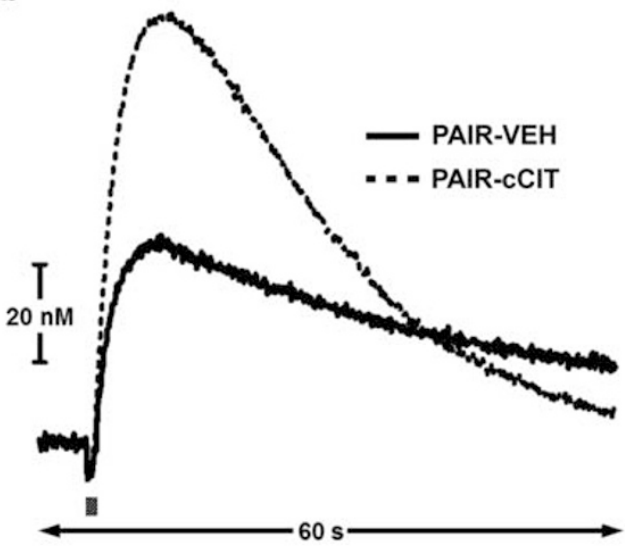

d

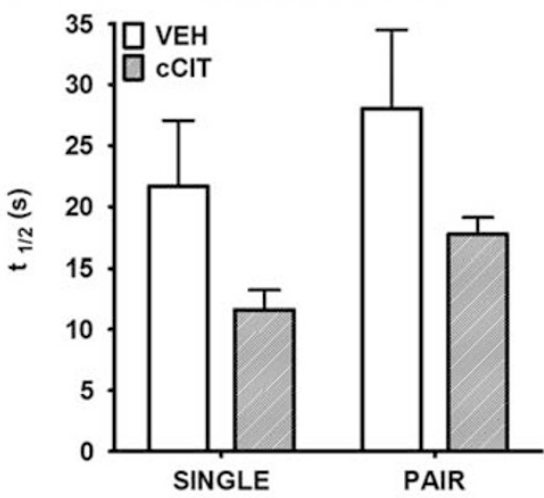

f Frequency dependence

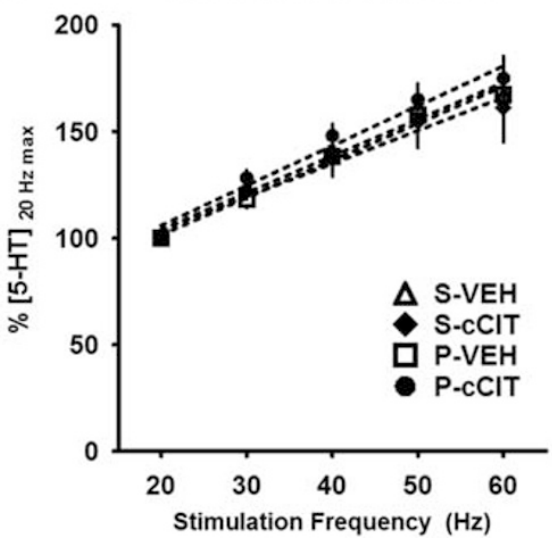

Figure 4 Comparison of electrically evoked serotonin release following acute CIT challenge. ( $a$ and $b$ ) Averaged concentration vs time traces of serotonin release evoked by a $60 \mathrm{~Hz}$, 60-pulse stimulation of the DRN $30 \mathrm{~min}$ after acute CIT. Shaded bar below trace indicates the duration of stimulation. (c) Mean rate of change in $[5-\mathrm{HT}]$ during the rising phase of release evoked by $60 \mathrm{~Hz}, 60$-pulse stimulation after acute $\mathrm{CIT}$ challenge. (d) Mean $t_{1 / 2}$ of evoked serotonin following $60 \mathrm{~Hz}, 60$-pulse stimulations. (e) Mean net overflow following $60 \mathrm{~Hz}, 60$-pulse stimulations. (f) Averages of normalized maximal release amplitudes evoked by $20-60 \mathrm{~Hz}$ stimulations. Dotted lines show best-fit linear regressions for each treatment group. Data shown are means \pm SEM. Tukey's multiple comparisons post hoc analysis: $* * P<0.01, * P<0.05$.

2013). Indeed, Davidson and Stamford showed that chronic paroxetine decreased autoreceptor function in the DRN, leading to enhancement of serotonin signaling, consistent with this proposed desensitization (Davidson and Stamford, 2000). Acute CIT administration results in rapid, reversible internalization of DRN 5-HT1a autoreceptors (Riad et al, 2004). The frequency-dependent effect of acute CIT exposure, such that observed in VEH-treated single- and pair-housed mice in Figure 4f, is also due to the effects of CIT on autoreceptors in the DRN (unpublished observations). Frequency-dependent signaling in cCIT-treated, pair-housed mice could arise from the suppression of autoreceptor feedback and would be a functional consequence of their desensitization. Even at low frequencies, cCIT substantively enhanced release in pair-housed mice after the acute CIT challenge. This indicates that during chronic treatment, 
baseline increases in release are intensified by ongoing uptake inhibition and may occur at low $(1-5 \mathrm{~Hz})$ firing rates. This increased release allows greater interaction of extracellular serotonin with its postsynaptic targets.

It could be hypothesized that chronic mild stress experienced by single-housed C57BL/6J mice would produce a depressive phenotype and intensify the effects of cCIT treatment when compared with VEH-treated counterparts. Surprisingly, social isolation blocked cCIT-induced facilitation of serotonin release, but did not induce changes in VEH-treated mice. Many behavioral and neurochemical effects of social isolation are correlated with an upregulation of HPA axis activity, including brain-wide increases in CRF and CORT levels (Backstrom and Winberg, 2013; Blanchard et al, 2001). One week of social isolation is sufficient to observe its anxiogenic effects (Conrad et al, 2011; Liu et al, 2013). This suggests that by the time the single-housed mice in this study began cCIT or VEH treatment, they were already experiencing consequences from the chronic mild stress paradigm. C57BL/6J mice are a resilient strain, and under ordinary conditions, these increases may not be behaviorally or neurochemically significant (Voikar et al, 2005). However, chronic SSRI treatment increases negative feedback to the HPA axis, reducing its function (Barden, 1996, 2004; Pariante and Miller, 2001). SSRI-induced changes in HPA axis activity directly affect serotonergic activity (Celada et al, 2001, 2004; Johnson et al, 2007; Kirby et al, 2008). Isolation-induced increases in HPA axis function could negate the opposing effects of chronic SSRI exposure. Thus, social isolation and CCIT treatment may work against each other so that serotonin signaling is not altered in single-housed mice.

Our finding that cCIT led to significant reductions in $\mathrm{MB}$ in the pair-housed, but not in the single-housed, mice provides additional evidence for lower sensitivity to SSRI effects with chronic social isolation (Figure 2a). Paradoxically, cCIT treatment had an anxiogenic effect in the OF test, decreasing center time in both single- and pair-housed mice (Figure 2d). SSRI treatments have variable effects on center time in other rodent studies, raising questions about the predictive validity of this measurement for anxiolytic effects of antidepressants (Borsini et al, 2002). As another example, environmental enrichment has also been shown to have generally anxiolytic effects in mice, but anxiogenic effects similar to ours are seen in the OF test (Branchi and Alleva, 2006; Whitaker et al, 2009). One interpretation is that these decreases in center time may indicate an adaptive increase in risk assessment, rather than enhanced anxiety. Thus, cCIT treatment may increase the ability of both single- and pairhoused mice to inhibit impulsive exploratory behaviors.

Plasticity in serotonergic function and its downstream targets underlies the efficacy of SSRI therapies for depression and OCD (Blier and de Montigny, 1998a). However, prior studies have not characterized the changes in endogenous serotonin dynamics that occur during the therapeutic period of SSRI treatment. In this study, we used in vivo measurements to show that 20-day cCIT treatment facilitates serotonin release in a frequency-dependent manner without altering SERT function. Prior studies have shown that autoreceptor desensitization occurs with the onset of therapeutic effects. Here, we demonstrate a consequence of this desensitization, revealing profound increases in evoked terminal serotonin release at all frequencies, a major mechanistic change that may contribute to the therapeutic actions of SSRIs. The observation that individually housing animals can entirely block this effect in both baseline and challenge conditions indicates that ongoing stress during SSRI treatment is an important variable in individual outcomes. This work shows that plasticity in the serotonergic system is sensitive to environmental stress, and this can influence the effects of SSRI therapies.

\section{FUNDING AND DISCLOSURE}

The authors declare no conflict of interest.

\section{ACKNOWLEDGEMENTS}

The authors thank Dr Rylan Larsen for assistance with confocal imaging and Nicholas Garcia for statistical expertise. We also acknowledge support from UNC Microscopy Core and the UNC Electronics Facility. This work was supported by an NIH grant to RMW (NS 015841) and by the UNC Intellectual and Developmental Disabilities Research Center, funded by NICHD (U54 HD079124).

\section{REFERENCES}

Arora T, Bhowmik M, Khanam R, Vohora D (2013). Oxcarbazepine and fluoxetine protect against mouse models of obsessive compulsive disorder through modulation of cortical serotonin and CREB pathway. Behav Brain Res 247: 146-152.

Backstrom T, Winberg S (2013). Central corticotropin releasing factor and social stress. Front Neurosci 7: 117.

Barden N (1996). Modulation of glucocorticoid receptor gene expression by antidepressant drugs. Pharmacopsychiatry 29: 12-22.

Barden N (2004). Implication of the hypothalamic-pituitaryadrenal axis in the physiopathology of depression. J Psychiatry Neurosci 29: 185-193.

Benmansour S, Cecchi M, Morilak DA, Gerhardt GA, Javors MA, Gould GG et al (1999). Effects of chronic antidepressant treatments on serotonin transporter function, density, and mRNA level. J Neurosci 19: 10494-10501.

Bespalov AY, van Gaalen MM, Gross G (2010). Antidepressant treatment in anxiety disorders. Curr Topics Behav Neurosci 2: 361-390.

Blanchard RJ, McKittrick CR, Blanchard DC (2001). Animal models of social stress: effects on behavior and brain neurochemical systems. Physiol Behav 73: 261-271.

Blier P, de Montigny C (1998a). Possible serotonergic mechanisms underlying the antidepressant and anti-obsessive-compulsive disorder responses. Biol Psychiatry 44: 313-323.

Blier P, Pineyro G, el Mansari M, Bergeron R, de Montigny C (1998b). Role of somatodendritic 5-HT autoreceptors in modulating 5-HT neurotransmission. Ann N Y Acad Sci 861: 204-216.

Borsini F, Podhorna J, Marazziti D (2002). Do animal models of anxiety predict anxiolytic-like effects of antidepressants? Psychopharmacology 163: 121-141.

Branchi I, Alleva E (2006). Communal nesting, an early social enrichment, increases the adult anxiety-like response and shapes the role of social context in modulating the emotional behavior. Behav Brain Res 172: 299-306.

Brandl EJ, Muller DJ, Richter MA (2012). Pharmacogenetics of obsessive-compulsive disorders. Pharmacogenomics 13: 71-81.

Bunin MA, Prioleau C, Mailman RB, Wightman RM (1998). Release and uptake rates of 5-hydroxytryptamine in the dorsal raphe and 
substantia nigra reticulata of the rat brain. $J$ Neurochem 70 : 1077-1087.

Celada P, Bortolozzi A, Artigas F (2013). Serotonin 5-HT1A receptors as targets for agents to treat psychiatric disorders: rationale and current status of research. CNS Drugs 27: 703-716.

Celada P, Puig M, Amargos-Bosch M, Adell A, Artigas F (2004). The therapeutic role of $5-\mathrm{HT} 1 \mathrm{~A}$ and 5 -HT2A receptors in depression. J Psychiatry Neurosci 29: 252-265.

Celada P, Puig MV, Casanovas JM, Guillazo G, Artigas F (2001). Control of dorsal raphe serotonergic neurons by the medial prefrontal cortex: Involvement of serotonin-1A, GABA(A), and glutamate receptors. J Neurosci 21: 9917-9929.

Chaouloff F, Berton O, Mormede P (1999). Serotonin and stress. Neuropsychopharmacology 21(2 Suppl): 28S-32S.

Conrad KL, Louderback KM, Gessner CP, Winder DG (2011). Stress-induced alterations in anxiety-like behavior and adaptations in plasticity in the bed nucleus of the stria terminalis. Physiol Behav 104: 248-256.

Dankoski EC, Wightman RM (2013). Monitoring serotonin signaling on a subsecond time scale. Front Integr Neurosci 7: 44.

Davidson C, Stamford JA (1997). Chronic paroxetine desensitises 5-HT1D but not 5-HT1B autoreceptors in rat lateral geniculate nucleus. Brain Res 760: 238-242.

Davidson C, Stamford JA (2000). Effect of chronic paroxetine treatment on 5-HT1B and 5-HT1D autoreceptors in rat dorsal raphe nucleus. Neurochem Int 36: 91-96.

de Montigny C, Pineyro G, Chaput Y, Blier P (1992). Electrophysiological studies on the effect of long-term 5-HT reuptake inhibition on the function of 5-HT neurons. Clin Neuropharmacol 15(Suppl $1 \mathrm{Pt} \mathrm{A}$ ): 440A-441A.

Dreyer JK, Herrik KF, Berg RW, Hounsgaard JD (2010). Influence of phasic and tonic dopamine release on receptor activation. J Neurosci 30: 14273-14283.

Fava M, Rush AJ, Alpert JE, Balasubramani GK, Wisniewski SR, Carmin CN et al (2008). Difference in treatment outcome in outpatients with anxious versus nonanxious depression: a $\mathrm{STAR}^{*} \mathrm{D}$ report. Am J Psychiatry 165: 342-351.

Ferres-Coy A, Santana N, Castane A, Cortes R, Carmona MC, Toth $\mathrm{M}$ et al (2013). Acute 5-HT(1)A autoreceptor knockdown increases antidepressant responses and serotonin release in stressful conditions. Psychopharmacology 225: 61-74.

Fredricson Overo K (1982). Kinetics of citalopram in test animals; drug exposure in safety studies. Prog Neuropsychopharmacol Biol Psychiatry 6: 297-309.

Gartside SE, Hajos-Korcsok E, Bagdy E, Harsing LG Jr, Sharp T, Hajos M (2000). Neurochemical and electrophysiological studies on the functional significance of burst firing in serotonergic neurons. Neuroscience 98: 295-300.

Gaynes BN, Rush AJ, Trivedi MH, Wisniewski SR, Spencer D, Fava M (2008). The STAR*D study: treating depression in the real world. Cleve Clin J Med 75: 57-66.

Hashemi P, Dankoski EC, Lama R, Wood KM, Takmakov P, Wightman RM (2012). Brain dopamine and serotonin differ in regulation and its consequences. Proc Natl Acad Sci USA 109: 11510-11515.

Hashemi P, Dankoski EC, Petrovic J, Keithley RB, Wightman RM (2009). Voltammetric detection of 5-hydroxytryptamine release in the rat brain. Anal Chem 81: 9462-9471.

Ichimaru Y, Egawa T, Sawa A (1995). 5-HT1A-receptor subtype mediates the effect of fluvoxamine, a selective serotonin reuptake inhibitor, on marble-burying behavior in mice. Jpn J Pharmacol 68: 65-70.

Jacobs BL (1991). Serotonin and behavior: emphasis on motor control. J Clin Psychiatry 52(Suppl): 17-23.
Jacobsen JP, Medvedev IO, Caron MG (2012). The 5-HT deficiency theory of depression: perspectives from a naturalistic 5-HT deficiency model, the tryptophan hydroxylase $2 \mathrm{Arg} 439 \mathrm{His}$ knockin mouse. Philos Trans R Soc Lond B Biol Sci 367: 2444-2459.

Jiao J, Nitzke AM, Doukas DG, Seiglie MP, Dulawa SC (2011). Antidepressant response to chronic citalopram treatment in eight inbred mouse strains. Psychopharmacology 213: 509-520.

Johnson DA, Grant EJ, Ingram CD, Gartside SE (2007). Glucocorticoid receptor antagonists hasten and augment neurochemical responses to a selective serotonin reuptake inhibitor antidepressant. Biol Psychiatry 62: 1228-1235.

Karelina K, DeVries AC (2011). Modeling social influences on human health. Psychosom Med 73: 67-74.

Kirby LG, Freeman-Daniels E, Lemos JC, Nunan JD, Lamy C, Akanwa A et al (2008). Corticotropin-releasing factor increases GABA synaptic activity and induces inward current in 5-hydroxytryptamine dorsal raphe neurons. J Neurosci 28: 12927-12937.

Liu X, Wu R, Tai F, Ma L, Wei B, Yang X et al (2013). Effects of group housing on stress induced emotional and neuroendocrine alterations. Brain Res 1502: 71-80.

Nestler EJ, Hyman SE (2010). Animal models of neuropsychiatric disorders. Nat Neurosci 13: 1161-1169.

Oreland L, Nordquist N, Hallman J, Harro J, Nilsson KW (2010). Environment and the serotonergic system. Eur Psychiatry 25: 304-306.

Pariante CM, Miller AH (2001). Glucocorticoid receptors in major depression: relevance to pathophysiology and treatment. Biol Psychiatry 49: 391-404.

Riad M, Zimmer L, Rbah L, Watkins KC, Hamon M, Descarries L (2004). Acute treatment with the antidepressant fluoxetine internalizes 5-HT1A autoreceptors and reduces the in vivo binding of the PET radioligand [18F]MPPF in the nucleus raphe dorsalis of rat. J Neurosci 24: 5420-5426.

Robinson DL, Wightman RM (2004). Nomifensine amplifies subsecond dopamine signals in the ventral striatum of freelymoving rats. J Neurochem 90: 894-903.

Shaskan EG, Snyder SH (1970). Kinetics of serotonin accumulation into slices from rat brain: relationship to catecholamine uptake. J Pharmacol Exp Ther 175: 404-418.

Strekalova T, Anthony DC, Dolgov O, Anokhin K, Kubatiev A, Steinbusch HM et al (2013). The differential effects of chronic imipramine or citalopram administration on physiological and behavioral outcomes in naive mice. Behav Brain Res 245: 101-106.

Uher R (2011). Genes, environment, and individual differences in responding to treatment for depression. Harv Rev Psychiatry 19: 109-124.

Valzelli L (1973). The 'isolation syndrome' in mice. Psychopharmacologia 31: 305-320.

Voikar V, Polus A, Vasar E, Rauvala H (2005). Long-term individual housing in $\mathrm{C} 57 \mathrm{BL} / 6 \mathrm{~J}$ and $\mathrm{DBA} / 2$ mice: assessment of behavioral consequences. Genes Brain Behav 4: 240-252.

Whitaker J, Moy SS, Godfrey V, Nielsen J, Bellinger D, Bradfield J (2009). Effects of cage size and enrichment on reproductive performance and behavior in C57BL/6Tac mice. Lab Anim 38: 24-34.

Wiborg O (2013). Chronic mild stress for modeling anhedonia. Cell Tissue Res 354: 155-169.

Wightman RM, Zimmerman JB (1990). Control of dopamine extracellular concentration in rat striatum by impulse flow and uptake. Brain Res Rev 15: 135-144.

Yorgason JT, Espana RA, Jones SR (2011). Demon voltammetry and analysis software: analysis of cocaine-induced alterations in dopamine signaling using multiple kinetic measures. Jneurosci met 202: 158-164.

Supplementary Information accompanies the paper on the Neuropsychopharmacology website (http://www.nature.com/npp) 